\title{
INFLUÊNCIAS DOS MÉTODOS DE COCÇÃO NA PRESERVAÇÃO DE ANTIOXIDANTES E COMPOSTOS BIOATIVOS, ANÁLISE FÍSICO-QUÍMICA E SENSORIAL EM ABÓBORA CABOTIÁ (Curbita Moschata Duch)
}

\section{COOKING DIFFERENT METHODS AND ITS INFLUENCE ON ANTIOXIDANT AND BIOACTIVE COMPOUNDS PRESERVATION, A PHYSICAL-CHEMICAL PUMPKIN (CURBITA MOSCHATA DUCH) ANAL YSES AND SENSORY EVALUATION}

\author{
Ana Souza De Paula ${ }^{1}$, Adriana Hernandes Martins ${ }^{2 *}$, Luiza Caroline Schlemer \\ Kramer $^{3}$, Juliana Aparecida Mirante Da Silva ${ }^{4}$

\begin{abstract}
${ }^{1}$ Nutricionista Centro Universitário Fundação Assis Gurgacz - FAG. ${ }^{2}$ Nutricionista Especialista, Universidade Tecnologica Federal do Paraná (UTFPR). ${ }^{4}$ Acadêmica Universidade Tecnologica Federal do Paraná (UTFPR).

*Autor correspondente: adrihernandesm@gmail.com, https://orcid.org/0000-0001-9718-5846
\end{abstract} \\ Docente do Curso de Nutrição, Centro Universitário Fundação Assis Gurgacz (FAG). ${ }^{3}$ Acadêmica
}

\section{RESUMO}

Introdução: A Cucurbitacea faz parte das famílias dos vegetais que são classificados em 2 gêneros como o Zanonioideae e a Cucurbitoideae, uma das classificações de hortaliças e frutas mais importantes do Brasil, com 100 gêneros e 825 espécies registradas, são representadas pelo melão, melancia, pepino e as abóboras. As Cucurbitas representam os frutos das abóboras, classificados cientificamente pelo seu formato, cor e textura da casca, se destacam pelo seu alto valor nutritivo e baixo teor calórico, além de serem fonte vitaminas, minerais, carotenoides com propriedades antioxidantes. Na Cucurbita Moschata Duch os compostos bioativos (CBAs) estão presentes em forma de Carotenos que atuam como percussores de vitaminas, compostos fenólicos, fatores antioxidantes e ainda recuperação de algumas patologias. Objetivo: $O$ presente estudo avaliará a preservação dos Compostos Bioativos (CBAs) na Cucurbita Moschata Duch Metodologia: submetidos a três diferentes métodos de cocção tradicionais: o fogão a gás (em vapor), forno microondas e na fritadeira elétrica (Air Fryer), com o objetivo de determinar a preferência da população através da análise sensorial com aplicação de um teste de ordenação, determinar o melhor método para preservação dos CBAs, atividade antioxidantes e composição físico-química da Cucurbita Moschata Duch. Resultados: O resultado obtido nesta pesquisa demostra que a cocção na Fritadeira Elétrica- Air Fryer preserva melhor os compostos fenólicos e atividade antioxidade da abóbora, já as análise físico-química demostra variedades e o resultado da análise sensorial demostrou que o método com melhor aceitabilidade foi o Microondas. Conclusão: Conclui-se que a cocção na Fritadeira Elétrica- Air Fryer é um método novo e não á registro de estudos sobre sua ação e efeito biológico do alimento após cocção neste equipamento, então a melhor forma de se consumir Cucurbita Moschata Duch é variar os métodos de cocção para garantia de saúde e absorção dos nutrientes e efeitos benéficos do alimento.

Palavras-chave: Cucurbitas. Métodos de Cocção. Compostos Bioativos. 


\begin{abstract}
Introduction: Cucurbitane comes from vegetables family which is classified in two genders, Zanonioideae and Cucurbitoideae, one of vegetables and fruits classifications most important in Brazil, with 100 genders and 825 register species, represented by melon, watermelon, pickles and pumpkins. Curcubit represents the pumpkins fruits, scientifically classified by its format, color and peel texture, highlighting for its high nutritive value and reduced in calories, additionally its power source of vitamins, minerals, carotenoids and its antioxidants. The bioactive compounds in Cucurbita Moschata Duch are available such as Carotens, which are vitamins, phenolic compounds, antioxidants precursors and acting positively with pathologies regeneration. Objective:This current study will evaluate the Cucurbita Moschata Duch bioactive compounds preservation which was subjected Methodology: by tree diverse traditional cooking methods: gas cooker (steam), microwave and Air Fryer, focusing to determine the population preference through sensorial analyses using the ordination test, aiming the best cooking method for bioactive compounds, antioxidants and physical-chemical Cucurbita Moschata Duch preservation. Final outcome shows that Air Fryer cooking method has been the best method to preserve the pumpkin phenolic compounds and its antioxidants. However, physical-chemical analysis shows variety and the sensory evaluation aimed that microwave cooking method has been the best one. Concluding, Air fryer is a new cooking method and there is no longer study about its positive biologic food effects, by this way emphasizing that varying different cooking method it's the best option to Results: consume Cucurbita Moschata Duch assuring the nutrient absorption and the food beneficial effects.
\end{abstract}

Keywords: Curcubit, Cooking methonds, Bioactive compounds.

\title{
1 INTRODUÇÃO
}

A Cucurbitacea faz parte das famílias dos vegetais que são classificados em 2 gêneros como o Zanonioideae e a Cucurbitoideae, uma das classificações de hortaliças e frutas mais importantes do Brasil, com 100 gêneros e 825 espécies registradas, são representadas pelo melão, melancia, pepino e as abóboras. As Cucurbitas representam os frutos das abóboras, classificados cientificamente pelo seu formato, cor e textura da casca, sendo as Cucurbita Maxima e Cucurbita Moschata as mais consumidas no país se destacam pelo seu alto valor nutritivo e baixo teor calórico, além de ser fonte vitaminas do complexo B (B1, B2 e B5), vitamina C, vitamina $E$, minerais, carotenoides provitamina $A$ com propriedades antioxidantes. (CATÁLAGO BRASILEIRO DE HORTALIÇAS, 2010; VERONEZI et al., 2012; SALES, et al.,2015).

A Cucurbita Moschata Duch, conhecida como Abóbora Cabotiá possui casca firme verde escura rugosa, sua polpa apresenta coloração amarelo alaranjada e suas sementes são beges claras, sua polpa contém a cada $100 \mathrm{~g}$ em média $0,2 \%$ de lipídeos, $1,3 \%$ de proteínas, $20 \mathrm{mg}$ de cálcio e $4,6 \%$ de carboidratos totais, vitamina A $540 \mathrm{mcg} / 100 \mathrm{~g}$. Por seu alto valor nutritivo, a Cucurbita Moschata Duch também apresenta atividades biológicas que previnem doenças cardiovasculares, cânceres, e ainda por ser rica em carotenoides pró vitamina A ajudam a reduzir índices de anemias, seus benefícios são extensos, a Abóbora Cabotiá desempenha um ótimo papel para indivíduos que estão em tratamento de redução de peso, tratamento de dislipidemia e diabetes tipo 2 por apresentar resistência a glicose sanguínea, 
controlando os níveis séricos. (CHOI et al., 2007. VERONEZI; JORGE, 2012. DAIUTO, et al., 2012).

Segundo Nishinho et al., (2002), o $\alpha$-caroteno e $\beta$-caroteno tem o efeito de exclusão sobre a tumorgênese, sendo que o a-caroteno tem um efeito superior comparado ao $\beta$-caroteno, ou seja estes carotenoides cessam o ciclo de multiplicação celular já no início da doença ou ainda na sua fase de propagação, por fim são os responsáveis pela pré- depositarem a vitamina A no organismo. Ainda em menores quantidades, estão presentes também na Cucurbita Moschata Duch a luteína, que é formada por uma molécula $\alpha$-caroteno e dois radicais hidroxilas, considerada potente antioxidante e por prevenir danos dos radicais livres nos tecidos. A luteína capta a luz azul que é considerada prejudicial, reduzindo em até $40 \%$ os riscos de danos macular, também previne aterosclerose, cataratas e alguns cânceres. (LADRUM et al., 2001; NISHINO et al., 2002; SILVA, 2004; AMBRÓSIO et al., 2006; CHOI et al., 2007; ÖTLES et al., 2007; RODRIGUEZ et al., 2008; JIANG, DU, 2011).

Os compostos fenólicos presentes nos alimentos desempenham diversos efeitos biológicos importantes, destacam-se pelo seu efeito protetor, contra doenças cardiovasculares, ajudam o sistema imune resposta inflamatórias, evitam tromboses e ajudam na vasodilatação, mas seu destaque se dá pela sua ação antioxidante. A atividade antioxidante através dos carotenoides desempenham uma função importante para o organismo, pois os antioxidantes têm a capacidade de prevenir ou evitar a oxidação de materiais facilmente oxidantes; sendo diversos substratos, moléculas simples, polímeros e biossistemas complexos, a atividade antioxidante inibem a formação de radicais livre e também podem eliminar radicais importantes para a propagação oxidativa, sendo assim interrompem a reação oxidativa. (NAMIKI, 1990; KAHKÖNEN et al.,1999; ANDREO, et al., 2006).

A maioria das tabelas químicas de composição dos alimentos apresenta os alimentos de forma crua, mas alguns alimentos tem a necessidade de passar pelo processo de cocção, durante a etapa de preparo e cocção, ocorre alterações de forma positiva ou negativa, como melhorar a capacidade de compostos naturalmente presentes no alimento, formação de compostos relacionado a atividade nutricional e perda de nutrientes. Segundo Scheibler et al., 2010, os fatores responsáveis pelas alterações químicas, físicas são determinados pelas diversas formas de cocção, tempo e temperatura. A maioria dos minerais e a Vitamina $C$ não são resistentes ao calor, ou seja, manter a permanência desses nutrientes é um grande desafio pois além de serem indispensáveis pelo organismo fazem parte de reações biológicas também importantes, no caso a atividade antioxidante. (BERNHARDTB et al., 2006; CAMPOS et al., 2008; SCHEIBLER et al., 2010).

O presente estudo avaliará a preservação dos antioxidantes, compostos bioativos (CBAs), análise físico-química e análise sensorial na Cucurbita Moschata Duch, Abóbora Cabotia, submetidos a três diferentes métodos de cocção tradicionais: o fogão a gás (em vapor); forno microondas, e na fritadeira elétrica (Air Fryer), com o objetivo de avaliar o melhor método para preservar os antioxidante, CBAs e comparar as análises físico-química nos três métodos de cocção Cucurbita Moschata Duch e concluir se o método de cocção mais aceito pela população se refere a melhor preservação do alimento.

\section{METODOLOGIA}

O presente trabalho, trata-se de uma pesquisa qualitativa e de comparação, aprovado pelo comitê de ética em um Centro Universitário particular de Cascavel- $\mathrm{Pr}$, 
a qual foi realizada com parceria com uma Universidade Tecnológica Federal do Paraná, na qual foram analisados os compostos bioativos da Cucurbita Moschata Duch (Abóbora Cabotia), adquiridos em um comércio de Cascavel - PR.

\subsection{PREPARO DA AMOSTRA}

As amostras foram testadas duas semanas antes da execução da análise sensorial, inicialmente foram realizados pré-testes de preparo, realizados teste de cortes, tempos e cocções diferentes, a fim de determinar o melhor corte, tempo e forma de cocção para realização da pesquisa. Após definição, foi realizada a compra das abóboras em um mercado local da Cidade de Cascavel- Pr.

No início do preparo para a análise sensorial e seleção das amostras, as abóboras já estavam higienizadas, descascadas e cortadas em corte palito padrão. Esta etapa foi realizada 6hrs antes de dar início a cocção, foram guardadas em sacos plásticos apropriados e em refrigeração de até $5^{\circ} \mathrm{C}$.

\subsection{MÉTODOS DE COCÇÃO}

Para comparações possíveis entre os 3 diferentes métodos de cocção, foram adotadas para a pesquisa, a cocção em um fogão a gás industrial da marca Steel de 6 fogareiros; cocção em um microondas modelo crisp - jet defrost 38 litros da Brastemp $\AA$, utilizado também fritadeira elétrica Mondial AF-03®) de 2,7 litros (Air Fryer) e fritadeira elétrica Cadence Perfect FRT531® de 2,3 litros (Air Fryer).

Foram monitorados tempo e temperatura dos preparos, a partir das elaborações dos 3 preparos de cocção seguindo o seguinte padrão: Cocção na Fritadeira Elétrica denominado número 1, Forno Microondas número 2, e cocção no Fogão a gás- à vapor número 3, totalizando 336 amostras (112 de cada método), o tempo de cocção foi monitorado por um relógio digital em todas as preparações para garantia de uma uniformidade em todas as análises sensoriais, a Cucurbita Moschata Duch foi servida em amostras de 15gramas.

\subsubsection{FRITADEIRA ELÉTRICA- AIR FRYER}

As abóboras foram colocadas até a metade do recipiente, que estava préaquecido, com 60 minutos iniciais com temperatura de $180^{\circ} \mathrm{C}$, a cada $15 \mathrm{~min}$ mexidas as Abóboras, e no fim do tempo foram colocados mais 15 minutos.

\subsubsection{FORNO MICROONDAS}

As abóboras foram colocadas em pirex de vidro e espalhados de forma uniforme e colocados na potência máxima do microondas e cozidas por 6 minutos finais.

\subsubsection{FOGÃO A GÁS- À VAPOR}

Inicialmente aquecida a água (cerca de 5l.), em uma panela inox e em outra panela também de inox colocado as abóboras cruas e fechado com tampa, estilo banho-maria, monitorado reabastecimento de água, a cocção foi feita com 35 minutos finais.

\subsubsection{SELEÇÃO DAS AMOSTRAS}

As amostras foram retiradas ao fim de cada método de cocção e organizadas com o porcionamento padrão de $100 \mathrm{~g}$ de cada amostra, sendo uma amostra de alimento cru, uma amostra do método de cocção em fritadeira elétrica - Air Fryer, uma amostra do método de cocção do forno microondas e uma amostra de cocção de 
fogão à gás- à vapor, foram todas armazenadas em sacos plásticos com embalagens metalizadas a vácuo e congelados em uma temperatura de $-10^{\circ} \mathrm{C}$ á $-30^{\circ} \mathrm{C}$ por 8 dias até serem encaminhados para os laboratórios de análises na Cidade de Cascavel- $\operatorname{Pr}$ e Toledo- Pr.

\subsection{ANÁLISE SENSORIAL}

Todos os participantes receberam orientações para ler o TCLE, Termo de Consentimento Livre Esclarecido, e assinar ao final aceitando a participar da pesquisa, receberam orientação quanto a individualidade de cada amostra e sua codificação contendo 3 números. Os participantes encaminhados para cabines de individuais, onde foi entregue o teste de ordenação por preferência, explicado para cada participante como deveria ser feito o preenchimento, conforme seu paladar individual de preferência, onde o primeiro campo referente à amostra de melhor preferência, 0 segundo campo referente à amostra mediada e o terceiro campo referente amostra de menor preferência. Por fim cada participante recebeu 3 amostras aleatórias de Cucurbita Moschata Duch (Abóbora Cabotia), talheres plásticos, guardanapo e água sendo orientados a beber a cada fim de amostra com intuito de limpar o palato.

\subsection{POPULAÇÃO DA AMOSTRA}

A população dos provadores da pesquisa foi constituída por indivíduos maiores de 18 anos de uma Universidade particular da cidade de Cascavel- Pr, e tiveram aceitação constada através da assinatura do TCLE (Termo de Consentimento Livre Esclarecido) que está em anexo como Apêndice A.

\subsection{ANÁLISE DE ESTATÍSTICA}

A tabulação de dados foi realizada em uma planilha de Excel, e para analisar os dados finais foram utilizados o método de ordenação bilateral, com critérios de teste de preferência aplicado por Friedman. Foram realizadas somatórias das ordenações obtidas através de uma fórmula especifica, através do resultado obtido pela fórmula, indicado qual o nível de significância, através do número de avaliadores obtido o valor tabelado, segundo tabela 35 de Christensen. (DUTCOSKY, S. D.; 2013)

$$
\mathrm{F}=\frac{12}{A v^{*} \mathrm{t}^{*}(\mathrm{t}+1)}{ }^{*}\left(\mathrm{~S} 1^{2}+\mathrm{S} 2^{2}+\mathrm{S} 3^{2}\right)-3^{*} \mathrm{Av}{ }^{*}(\mathrm{t}+1)
$$

Tabela de Christensen 35- Valor Tabelado $(a)=29$. Nível de significância $=5 \%$

\subsection{ANÁLISES DE COMPOSTOS FENÓLICOS E FATOR ANTIOXIDANTES}

As análises foram realizadas em um laboratório com técnicos especializados na análise, em uma Universidade Tecnológica Federal da cidade de Toledo-Pr. Todos os métodos foram realizados por triplicata. Para a determinação de compostos fenólicos, o método utilizado segundo Singleton e Rossi (1965), primeiramente as amostras passaram por uma extração do composto fenólico que é realizada por um solvente a base de cetona, após finalização da extração passam a serem filtrados e por fim adicionado uma porcentagem de ácido gálico junto a amostra, ao chegar em temperatura ambiente as amostram foram determinas pela absorbância á temperatura média por espectrofotômetro (BOROSKI et al., 2015).

Para a determinação de atividade antioxidante, utilizado o método DPPH segundo Bondet (1997), para a determinação de DPPH, foram utilizados um extrato etanólico de todas as amostras analisadas, adicionados ao DPPH e preparado um 
tubo somente de DPPH sem amostra, todas as amostras aguardaram por $90 \mathrm{~min}$. longe de luz, por último foram determinadas através da absorbância por espectrofotometria (BOROSKI et al., 2015).

\subsection{ANÁLISE FÍSICO-QUÍMICA}

As análises Físico-Químicas foram realizadas no laboratório da Instituição Fundação para o Desenvolvimento Científico e Tecnológico- FUNDETEC, localizado na cidade de Cascavel-Pr. As análises foram preparadas e realizadas pela autora acompanhada de um técnico especializado onde o carboidrato será calculado por diferença, as proteínas segundo Kjeldahl, os lipídeos pelo método Soxhlet, as cinzas pela mufla e a umidade por estufa $550^{\circ} \mathrm{C}$ segundo a metodologia do Instituto Adolfo Lutz (2008).

\section{RESULTADOS E DISCUSSÃO}

Foram realizadas 112 análises sensoriais com indivíduos não treinados, os critérios de exclusão foram informados aos participantes, foram excluídos os testes de avaliadores com preenchimento incompleto ou preenchimento errado. Após tabulação de 112 amostras, segundo a tabela 35 de Christensen, o valor tabelado é 29 , no qual explica que o valor maior ou igual ao tabelado significa que apresenta diferença entre os métodos de cocção, já as resultado da somatória do teste de ordenação de preferência explicam que o maior resultado significa o método de cocção com maior rejeição e o menor com maior aceitabilidade pela população. Conforme demonstra o gráfico na figura 1.

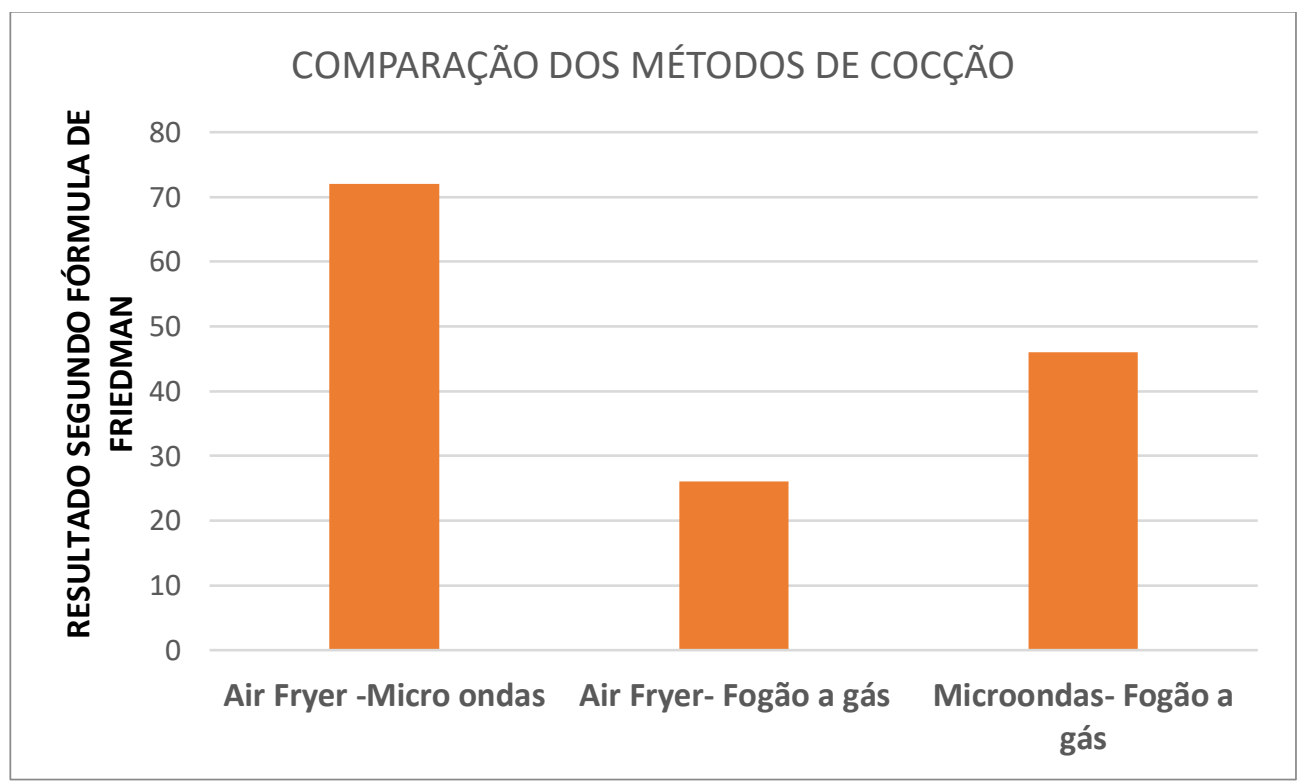

Figura 1. Comparação entre os métodos de cocção

Segundo análise estatística do resultado da análise sensorial da Cucurbita Moschata Duch obteve valor de 15,75 de acordo com o resultado da fórmula de Friedman, considerando a relação do nível de significância de $5 \%$. As comparações entre a amostra do método da Fritadeira Elétrica Air Fryer e amostra do Fogão a gása vapor apresentou resultado acima do tabelado sendo consideradas diferentes entre 
si; já a comparação entre amostra do método Fritadeira Elétrica Air Fryer e amostra do métodos Micro-ondas apresentaram resultado abaixo do tabelado consideradas estatisticamente sem diferença entre si e por último a comparação entre as amostras do métodos Micro-ondas e Fogão a gás a vapor apresentou o resultado acima do tabelado, assim sendo consideradas diferentes entre si. O método que se mostrou com maior índice de rejeição pelo teste de ordenação de preferência foi a Fritadeira Elétrica- Air Fryer; o de aceitação mediada foi o método de cocção no Fogão a gás- à vapor, e o mais aceito e preferido pelos indivíduos foi o microondas.

Os métodos de cocção Air-Fryer e Fogão a gás a vapor se demostram os mais rejeitados segundo o resultado da tabulação do teste de preferência demonstrado pelo gráfico 2 e não se diferenciam entre si segundo a comparação dos métodos de cocção apresentados, já o método de cocção do Micro-ondas apresentou o método mais aceito e diferentes entre si, em relação aos outros métodos de cocção, pela população da pesquisa. Segundo Scheibler et al. (2010) as diferentes formas de preparo, transferência de calor, duração de toda a cocção são consideradas influentes nas alterações físicas e químicas dos alimentos, mas deve-se considerar alterações desde o cultivo do alimento até o preparo das amostras, sendo assim todo e qualquer tipo de alteração interfere no paladar do indivíduo, este conjunto se torna fundamental ao se determinar a preferência do produto.

O INSTITUTO ADOLFO LUTZ, (2008) explica que a análise sensorial tem a função de demostrar através das respostas adquiridas pela análise, utilizando-se todos os seus sentidos como a visão, o olfato, a audição, o tato e o paladar, juntas podem trazer aos indivíduos participantes suas memórias afetivas, relacionando sua memória com a avaliação sensorial.

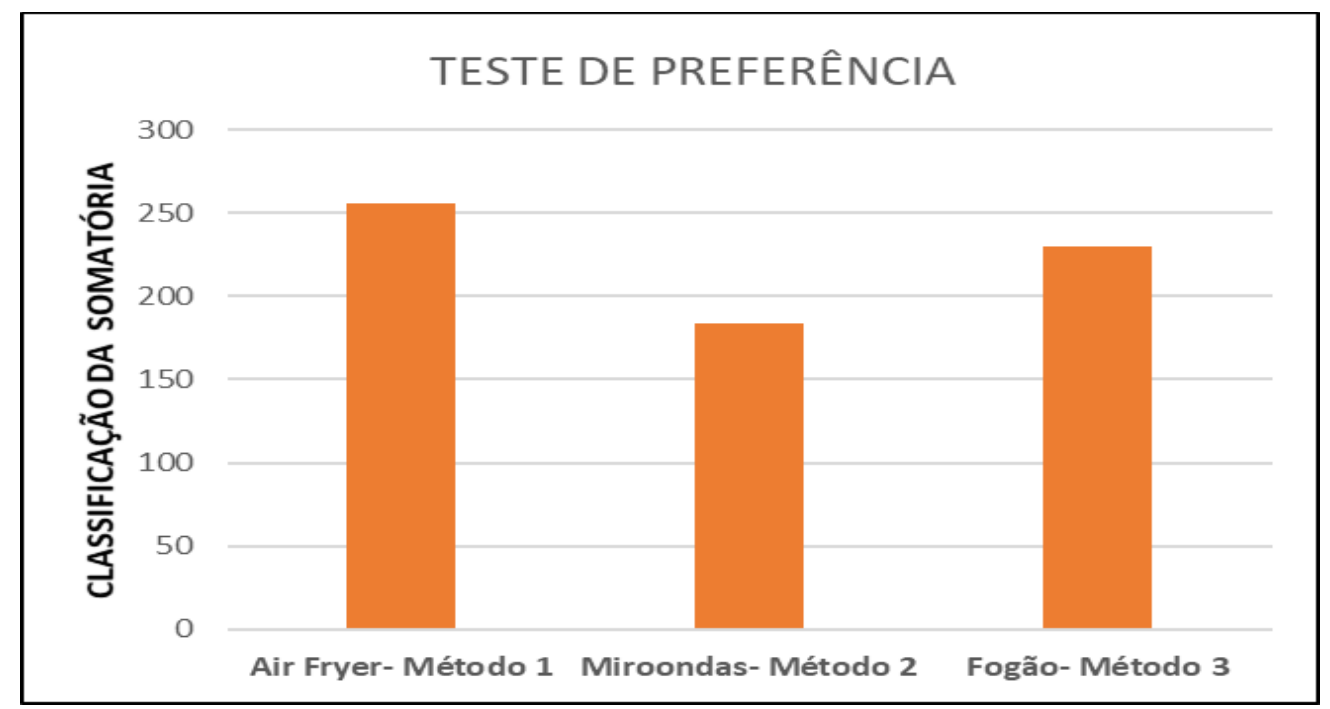

Figura 2. Somatória da análise sensorial- Teste de preferência

Entre as amostras analisadas, a Air Fryer mostrou ser um método de difícil aceitabilidade pelo fato de alterar fisicamente o alimento, a cocção ocorre por um calor seco em alta temperatura, resultando em um alimento mais seco devido a sua desidratação, já a aparência ficou envelhecida e escura alterando também seu aroma e sabor. O grau de cozimento, combinados pelo tempo e temperatura de aquecimento, não atua somente sobre a eliminação de microrganismos e enzimas, mas também acarreta em modificações nas suas propriedades organolépticas do produto cozido, alguns estudos sugerem que o cozimento realizado em forno micro-ondas provoca 
menos perdas no alimento, por ser realizado em menos tempo comparado a outros métodos de cocção (SCHEIBLER et al., 2010; DAIUTU et al. 2012).

Os compostos fenólicos presentes no alimento são facilmente oxidáveis, provocadas pelas enzimas presentes nos alimentos, e pela presença de metais, luz e o calor, provocando o escurecimento enzimático do alimento. Os compostos fenólicos funcionam como sequestradores de radicais livres, portando são eficazes na prevenção da oxidação lipídica, já para o alimento, os compostos fenólicos atribuem a cor, textura, amargor e adstringência do alimento (SIMÕES, 2001).

A amostra do método de cocção da Air Fryer demostrou um escurecimento enzimático, alteração de aroma e sabor da Abóbora, provocada pelos fenólicos presentes ao alimento após serem expostos ao método de cocção da Fritadeira Elétrica- Air Fryer. O resultado da análise de compostos fenólicos da Cucurbita Moschata Duch, mostrou que a maior concentração de fenólicos no alimento foi após o método da Air Fryer, o método do microondas e fogão também tem uma presença significativa de fenólicos e não são diferentes entre si, como mostra o gráfico abaixo.

O alimento Cru mostrou uma concentração menor de compostos fenólicos, comparada aos métodos de cocção, conforme o Gráfico na figura 3, deve-se considerar que alguns alimentos têm maior ação de compostos fenólicos após a etapa de cocção devida à concentração ou diluição de sua composição, como no caso da abóbora.

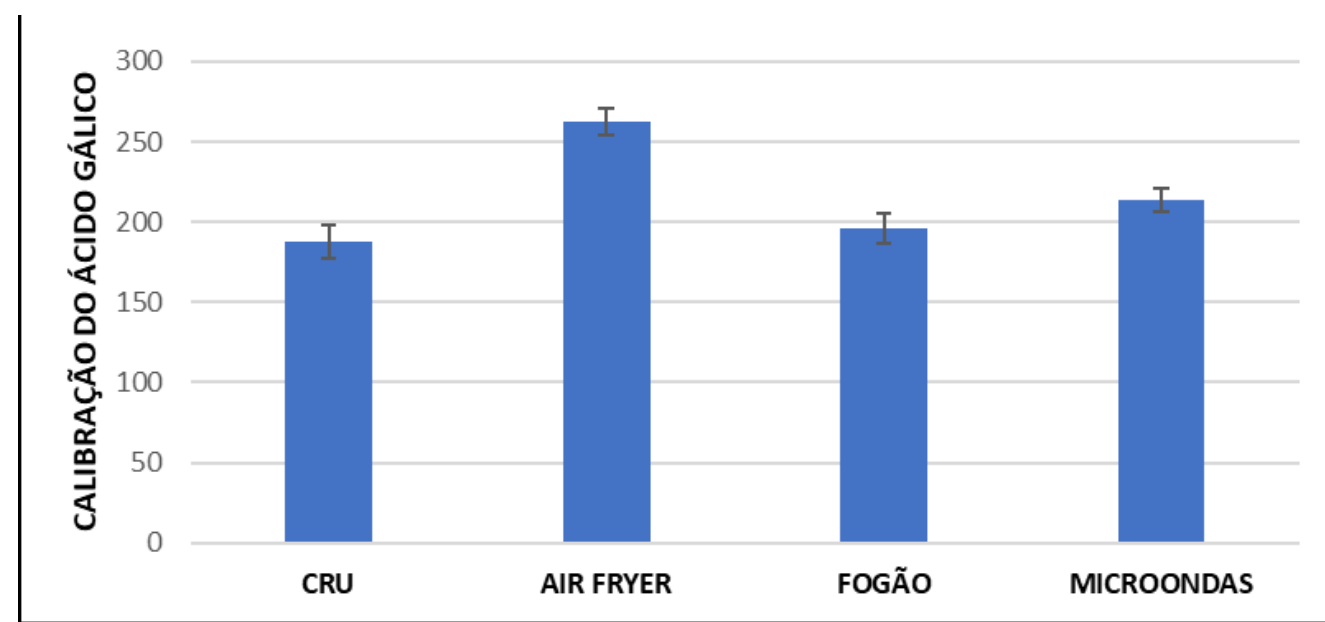

Figura 3. Comparação de compostos fenólicos

Segundo Bailey (1996) a atividade antioxidante apresenta alguns efeitos positivos, como baixo índice de atividade indesejáveis no alimento, sendo a oxidação não tóxica, ainda preserva cor, odor e sabor do alimento. Os carotenoides são os responsáveis pela atividade antioxidativa da Cucurbita Moschata Duch, e são classificados como primários e secundários desempenhando atividades importantes para o alimento e o indivíduo. Sendo um importante protetor contra microrganismos e patógenos na planta e para o individuo previne contra diversas patologias. O processo que o alimento passa, desde o cultivo até sua apresentação final, acarreta em diversas transformações, estas podem ser consideradas de ganho ou percas de seus nutrientes (ANTUNES, 1999; CERQUEIRA et al., 2007).

A atividade antioxidante se demostrou melhor no método de cocção da Air Fryer, sendo comparada a amostra crua e os demais métodos de cocção, onde a 
cocção foi feita por calor seco a $180^{\circ} \mathrm{C}$, como pode ser observar no gráfico na figura 4.

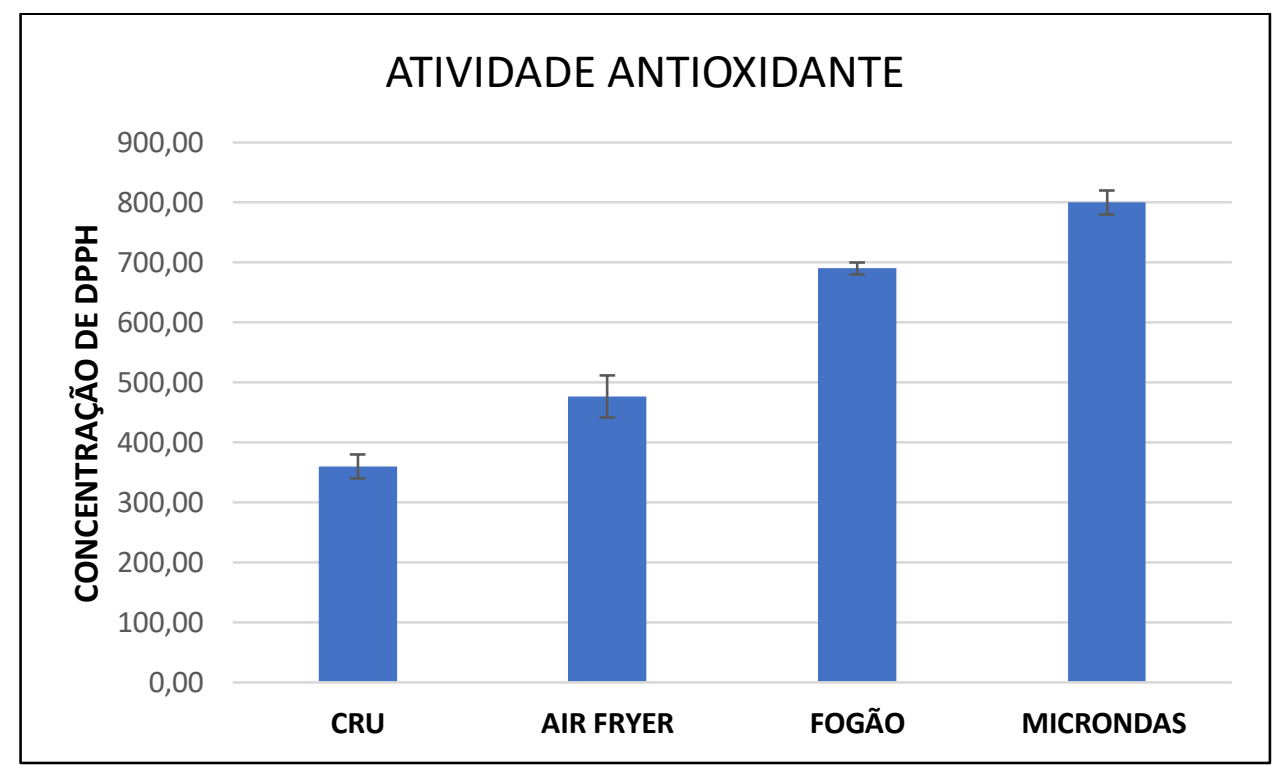

Figura 4. Comparação de Atividade Antioxidante

O resultado do alimento cru demostrou maior atividade antioxidante, mas a Cucurbita Moschata Duch é consumida somente após etapa de preparo, a forma de cocção são fatores determinantes para a conservação de atividades biológicas benéficas aos indivíduos e segundo a análise o melhor método é Air Fryer, por segundo o Fogão e o Microondas se demostrou o pior método para atividade oxidativa.

Os métodos de cocção têm uma grande influência quanto a preservação físicoquímica do alimento, o conhecimento dos principais fatores que influenciam o ganho ou perca destes nutrientes torna possível prevenir ou até mesmo reduzir essas perdas nutricionais. (BERNHARDT E SCHLICH, 2006). Os métodos de preparo e cocção das amostram foram bem distintos entre si, não foram consultados uma referência científica de melhor método de cocção para se consumir vegetais, entre tanto todas as amostras foram levadas para uma análise físico-química e os resultados encontram-se na Tabela 1.

Tabela 1. Valores encontrados (\%) - análises físico-químicas

\begin{tabular}{cccccc}
\hline AMOSTRA & UMIDADE & PROTEÍNA & LIPÍDIOS & CINZAS & CARBOIDRATOS \\
\hline Fogão & 85,76 & 2,44 & 0,19 & 1,13 & 10,47 \\
Micro-ondas & 84,22 & 2,11 & 0,12 & 1,23 & 12,32 \\
Air fryer & 70,72 & 4,73 & 0,19 & 2,53 & 21,82 \\
Crua & 88,00 & 2,31 & 0,27 & 0,98 & 8,44 \\
\hline
\end{tabular}

Fonte: Dados fornecidos pela Instituição Fundetec, 2018.

O resultado desta pesquisa de análise físico-química para umidade, demostrou que o método que utilizou Fogão a gás com aplicação de calor úmido e Micro-ondas se comportaram de forma semelhante a preservação da umidade quando comparada a amostra crua, já o método em Air Fryer demostra que a cocção deste equipamento ocorre em calor seco desidratando o alimento.

Entre os métodos analisados, a Fritadeira Elétrica Air Fryer se demostrou com maior porcentagem de cinzas devido ao baixo valor de umidade como mostra a tabela 
anterior. O método de cocção aplicado à Air Fryer é calor seco, sendo assim melhor método para se conservar resíduos sólidos.

Contrário aos valores encontrados nas amostras preparadas em fritadeira Airfryer e forno micro-ondas, a cocção por calor úmido em fogão a gás apresentou $1,13 \%$ de cinzas, sendo esta a maior perda de minerais entre os métodos. Scheibler et al. (2010) relataram em seu estudo que, quando um alimento de origem vegetal é cozido em água e não é ultrapassado o tempo necessário para sua cocção, a concentração de seus minerais tende a não sofrer alterações significativas. Em complemento, o mesmo estudo evidencia que, quando métodos de cocção incitam a desidratação do alimento, ocorre a concentração de seus minerais devido à perda de água, o que pode justificar a elevação das porcentagens de cinzas das demais amostras.

Com relação a proteína, um dos principais componentes celular, tem diversas funções no organismo humano dentre elas a sustentação da célula através do colágeno e da elastina e influencia diretamente em alguns processos biológicos importantes pois incluem enzimas capazes de fazer a canalização de várias reações químicas que ocorrem no intestino. (MARZZOCO, et al., 1999)

Dos métodos aplicados nesta pesquisa o que demostra maior concentração de proteína é a Fritadeira Elétrica Air Fryer, os outros métodos apresentaram resultados inferiores, porém vale ressaltar que não foram analisadas a biodisponibilidade desta proteína ao organismo humano.

O carboidrato presente nos alimentos são responsáveis por fornecer energia ao organismo, serve como principal componente da membrana celular que intermedia as comunicações intracelulares, os carboidratos são os responsáveis, na maioria das vezes, pelo sabor adocicado dos alimentos. (CHAMPE e HARVEY; 1996).

Segundo o resultado da análise, os carboidratos das amostras após cocção apresentaram resultados maiores que na forma do alimento cru, o de melhor resultado foi o método de cocção da Air Fryer seguido do micro-ondas, os carboidratos são de extrema importância em uma dieta pois ocupam até $50 \%$ de sua composição.

Os lipídeos presentes em uma dieta são definidos como gorduras, fazem parte da absorção de vitaminas lipossolúveis, se consumida de forma correta não apresenta risco a saúde humana. Abaixo o gráfico da preservação de lipídeos:

A amostra crua demostrou maior concentração de lipídeo, mas a abóbora cabotiá é um vegetal que necessita passar pela etapa de cocção para ser consumida. Por tanto os método do Fogão a gás e Air Fryer apresentaram resultados parecidos sendo assim considerados bons métodos para a conservação de lipídeo da Cucurbita Moschata Duch.

Tem-se então, que a cocção em vapor do fogão a gás não apresentou resultados positivos no que se refere ao teor de carboidratos, proteínas e resíduos minerais, e que a preservação de umidade encontrado deve-se ao acréscimo de água utilizado no preparo da amostra. Enquanto o forno micro-ondas apresentou aspectos de estabilidade por não gerar grandes alterações, para mais e para menos, nos teores de umidade, cinzas e macronutrientes das amostras. Em oposição a isso o tratamento térmico em fritadeira Airfryer que causa a desidratação da amostra, apresentou o aumento de todos dos critérios avaliados na análise físico-química, com exceção apenas para o teor de umidade por este ser justamente o motivo da desidratação e concentração dos componentes das amostras, representados pelo aumento nos teores de cinzas, carboidratos e proteínas.

A preservação dos nutrientes analisados neste trabalho da Cucurbita Moschata Duch mostrou em maior concentração no método de cocção Air Fryer, mas devemos 
considerar que não existem estudos aprofundados deste equipamento, ou seja, não foram analisados a biodisponibilidade dos nutrientes analisados e danos que 0 equipamento pode causar ao organismo humano.

\section{CONCLUSÃO}

Os resultados mostram que os métodos de preparo e cocção interferem de forma significativa na concentração de compostos fenólicos, atividade antioxidante e concentração físico-química do alimento, sendo que alguns alimentos necessitam passar por uma etapa de cocção para a atividade antioxidante ser melhorada.

Os diferentes métodos comparados demostraram que o método de preferência da população não se mostrou com melhor composição fenólica, atividade antioxidante e ainda maior concentração de carboidrato, proteína, lipídio, cinzas e umidade, mas que a escolha partiu da apreciação visual, odor e sabor das amostras. O método de cocção da Air Fryer denominou-se com os melhores resultados de compostos fenólicos e atividade antioxidante, mas apresento maior rejeição pela população, comprovando que a memória afetiva, apresentação visual, sabor e odor do alimento são determinantes na aceitabilidade.

Conclui-se que diante do resultado obtido através do estudo, que o consumo da abóbora deve ser realizado em seus diferentes e possíveis métodos de cocção, podendo aproveitar o benefício nutricional e biológico que alimento apresenta.

\section{REFERÊNCIAS BIBLIOGRÁFICAS}

ANDREU, S. A. et al. Mathematical quantification of total carotenoids in Siomaß oil using color coordinates and multiple linear regression during deep-frying simulations. European Food Research and Technology, v. 6, n.226, p. 1283-1291, 2006.

AMBRÓSIO, C. L. B. et al. Carotenóides como alternativa contra a hipovitaminose A. Rev. Nutr. vol.19 n.2, Campinas. 2006.

ANTUNES R. Os sentidos do trabalho: ensaio sobre a afirmação e a negação do trabalho. São Paulo: Boitempo, 1999.

BERNHARDT, S.; SCHLICH, E. Impact of different cooking methods on food quality: retention of lipophilic vitamins in fresh and frozen vegetables. Journal of Food Engineering, Amsterdam, v. 77, n. 1, p. 327-333, nov. 2006.

BOROSKI, M., et al., Livro: Antioxidantes: princípios e métodos analíticos. 1. Ed.Curitiba, Appris, 2015. ISBN 978-85-8192-730-5.

CAMPOS, M. F. et al. Determinação dos teores de vitamina C em Hortaliças Minimamente Processadas. Revista Alimentos e Nutrição -2008.

CATÁLOGO BRASILEIRO DE HORTALIÇAS. Embrapa/ SENAE, Brasília- DF- 2010 SCHEIBLER, J. et al. Quantificação em micronutrientes em vegetais submetidos a diferentes métodos de cocção para doente renal crônico. ConsScientiae Saúde, São Paulo, v. 9, n. 4, p. 549-555, 2010. 
CERQUEIRA, P. M. et al. Efeito da farinha de semente de abóbora (Cucurbita maxima, L.) sobre o metabolismo glicídico e lipídico em ratos. Revista de Nutrição, v. 21, n. 2, p. 129-136, 2008.

$\mathrm{CHOI}, \mathrm{H}$. E. O. et al. Watersoluble extract from Cucurbita moschata shows antiobesity effects by controlling lipid metabolism in a high fat diet-induced obesity mouse model.

Biochemical and Biophysical Research Communications, 359; 419-425, 2007.

DAIUTOA, R. É. Et al. Alterações Nutricionais em casca e polpa de abóbora decorrentes de diferentes métodos de cozimento. Revista lberoamericana de Tecnología Postcosecha- 2012.

KAHKÖNEN et al. Antioxidant activity of plant extracts containing phenolic compounds. Journal of Agricultural and Food Chemistry, v.47, p.3954-3962, 1999.

LANDRUM, J. T.; BONE, R. A. Lutein, zeaxanthin and the macular pigment. Archives Biochemistry and Biophysics, New York, v. 28, n. 1, p. 28-40, Jan. 2001.

MARZZOCO, A. T., et al. Bioquímica Básica. $2^{\circ}$ Edição. Editora Guanabara Koogan, 1999

NAMIKI, M. Antioxidants/antimutagens in food. Journal of Nutrition, Boca Raton, v.29, n.4, p.273-300, 1990.

SIMÕES, C. M.C., et al. Farmacognosia: da planta ao medicamento. 3 ed. Porto Alegre/ Florianópolis: Ed. UFSC/ Ed. UFRG, 2001. 883p.

NISHINO, H. et al. Carotenoids in cancer chemoprevention. Cancer and Metastasis Reviews, v. 21, n. 4, p. 257-264, jun. 2002.

ÖTLES, S.; ÇAGINDI, Ö. Carotenoids as natural colorants. In: SOCACIU, C. Food colorants: chemical and function properties. Washington: CRC Press, 2007. p. 51-70.

O INSTITUTO ADOLFO LUTZ, (2008).

RODRIGUEZ, A., et al. Fontes brasileiras de carotenoides: tabela brasileira de composição de carotenóides em alimentos. 1ํㅡ reimpressão, 2009. Brasília, 2008.

SALES, L. A. M., et. al. Potencial das Sementes de Abóbora Submetidas a Diferentes Períodos de Embebição. Brazilian Journal of Biosystems Engineering v. 9(4): 289-297, 2015.

SILVA, O. C; et al., Livro: Ciência dos Alimentos- Princípios de Bromatologia. 1. Ed. Rubio- RJ, 2006. ISBN 978-85-8411-070-4.

SILVA, A.S. Luteína, alimento para tu vista. Food Ingredients. São Paulo, v. 6, n. 1, p. 80-81, jul. 2004. 
SCHEIBLER et al., Quantificação de micronutrientes em vegetais submetidos a diferentes métodos de cocção para doente renal crônico. ConScientiae Saúde2010

VERONEZI, C.M.; JORGE, N. Carotenoides em abóbora. Boletim do Centro de Pesquisa e Processamento de Alimentos (CEPPA), Curitiba, v. 29, n. 1, 2011. 